\title{
IPSC-MSC inhibition assessment in Raw 264.7 cells following oxygen and glucose deprivation reveals a distinct function for cardiopulmonary resuscitation
}

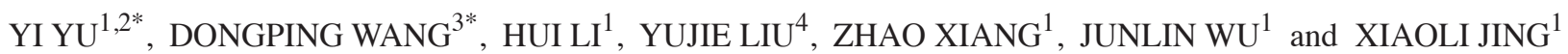 \\ ${ }^{1}$ Emergency Department, The First Affiliated Hospital of Sun Yat-sen University, Guangzhou, Guangdong 510080; \\ ${ }^{2}$ Department of Critical Care Medicine, Guangdong Provincial Hospital of Traditional Chinese Medicine, Guangzhou, \\ Guangdong 510006; ${ }^{3}$ Organ Transplant Center, The First Affiliated Hospital of Sun Yat-sen University; \\ ${ }^{4}$ Department of Breast Surgery and Key Laboratory of Malignant Tumor Gene Regulation and \\ Target Therapy of Guangdong Higher Education Institutes, Sun Yat-Sen Memorial Hospital, \\ Sun Yat-Sen University, Guangzhou, Guangdong 510080, P.R. China
}

Received March 10,2017; Accepted November 2, 2017

DOI: $10.3892 / \mathrm{mmr} .2018 .8864$

\begin{abstract}
Hypoxia is a serious stress state. The nervous system is less tolerant to hypoxia, and cell death due to hypoxia is irreversible. With the incidence of cardiovascular disease gradually increasing, the sudden cardiac death rate is additionally increasing. Although cardiopulmonary resuscitation (CPR) is an important development, recovery is frequently poor. In a successful recovery population, $\sim 40 \%$ of the population was in a vegetative state or subsequently succumbed to their condition, and $20 \%$ had brain damage. Therefore, the recovery of the brain is of particular importance in CPR. Immune disorders are one of the major mechanisms of cerebral resuscitation following CPR. Studies have demonstrated that induced pluripotent stem cell-derived mesenchymal stem cells (IPSC-MSCs) have a strong immune regulatory effect during tissue repair and anti-inflammatory effects. IPSC-MSCs may inhibit the inflammatory response by means of the inflammatory reaction network to improve brain function following CPR, although the cellular and molecular mechanisms remain unclear. Macrophages are a bridge between innate immune and specific immune responses in the body; therefore, it was hypothesized that macrophages may be the important effector cell of the role of IPSC-MSCs in improving brain function
\end{abstract}

Correspondence to: Professor Xiaoli Jing, Emergency Department, The First Affiliated Hospital of Sun Yat-sen University, 58 Zhongshan Second Road, Guangzhou, Guangdong 510080, P.R. China

E-mail: jing-xl@163.com

*Contributed equally

Key words: induced pluripotent stem cell-derived mesenchymal stem cells, cardiopulmonary resuscitation, oxygen and glucose deprivation, immune disorder, cerebral resuscitation following recovery of spontaneous respiration and circulation subsequent to cardiopulmonary resuscitation. In the present study, IPSC-MSCs were applied to the oxygen and glucose deprivation (OGD) model. It was observed that intervention with IPSC-MSCs was able to alter the polarization direction of macrophages. The difference in the proportions of M1 and M2 macrophages was statistically significant at 6, 12, 24 and $48 \mathrm{~h}(\mathrm{P}=0.037, \mathrm{P}<0.05)$ in the OGD + IPSC-MSCs group (M1, $33.48 \pm 5.6 \%$; M2, 50.84 $\pm 6.9 \%$ ) and in the OGD group (M1, $83.55 \pm 7.3 \%$; M2, $11.41 \pm 3.2 \%$ ), and over time this trend was more obvious. The polarization direction of macrophages is associated with the neurogenic locus notch homolog protein 1 (Notch-1) signaling pathway. In conclusion, it was observed that IPSC-MSCs may be associated with altered macrophage polarization, which may be accomplished by inhibiting the Notch-1 signaling pathway.

\section{Introduction}

Hypoxic encephalopathy caused by sudden cardiac death (1) is a critical clinical disease, and it is an important reason for the high rate of disability following cardiopulmonary resuscitation (CPR). Early identification, effective prevention, treatment of ischemic encephalopathy and improving the success rate of cerebral resuscitation are important issues that may not be ignored.

At present, studies have demonstrated that there are various reasons for the low success rate of treatment for hypoxic encephalopathy. During and subsequent to cardiopulmonary resuscitation, high levels of circulating cytokines, the activation of blood coagulation and platelets, the presence of endotoxins in plasma, and the alteration of soluble E-selectin and P-selectin have been described (2). A considerable number of studies have demonstrated that induced pluripotent stem cell-derived mesenchymal stem cells (IPSC-MSCs) have a marked therapeutic effect on hypoxic encephalopathy (3). IPSC-MSCs have exerted substantial protective effects and improvements on the survival rate of acute lung injury in animal 
experiments (4-6). In addition, intervention with IPSC-MSCs may reduce neutrophil infiltration in the lung tissue of mice with ventilator-associated pneumonia and improve the survival rate $(7,8)$. All of the above studies have demonstrated that IPSC-MSCs exert an immune regulatory effect and an inflammatory response that balances multiple aspects of the immune inflammatory network of the body; however, the details of the function of IPSC-MSCs and the mechanism of action remain unclear $(9,10)$. The role of macrophage differentiation, different phenotypes and functional status in inflammatory and neoplastic diseases has attracted much attention. Previous studies have demonstrated that the Notch-1 signaling pathway is associated with the differentiation, proliferation and function of a number of types of immune cells $(11,12)$. However, the mechanisms underlying the way in which IPSC-MSCs exert their benefits are not well understood.

IPSC-MSCs may improve the recovery of the brain following CPR; however, the mechanism underlying the role of IPSC-MSCs in immune regulation, whether they are able to alter the direction of macrophage polarization, and whether they may improve the prognosis of cerebral resuscitation remains unknown. Further experimental studies are required to examine the mechanism underlying the way in which IPSC-MSCs exert their anti-inflammatory effect, and which signaling pathway results in the induction of M2 type macrophages. In the present study, Raw 264.7 cells were used to perform oxygen and glucose deprivation (OGD) to replicate the model of cerebral ischemia. Intervention by IPSC-MSCs was performed in the OGD model and the results demonstrated that IPSC-MSCs were able to regulate the polarization of macrophages via the neurogenic locus notch homolog protein 1 (Notch-1) signaling pathway. In addition, the results of the present study demonstrated that IPSC-MSCs were able to regulate the polarization of macrophages, which may be accomplished by inhibiting the Notch-1 signaling pathway.

\section{Materials and methods}

Materials. Cells, cell culture media, serum and cell culture supplements were purchased from the American Type Culture Collection (Manassas, VA, USA), EMD Millipore (Billerica, MA, USA), Invitrogen (Thermo Fisher Scientific, Inc. Waltham, MA, USA), and PAA Laboratories (GE Healthcare, Chicago, IL, USA), respectively, unless otherwise stated. Antibodies against inducible nitric oxide synthase (iNOS; cat. no. ab178945), Hes1 (cat. no. ab108937), interleukin (IL)-10 (ab189392), arginase-1 (Arg1; cat. no. ab124917) and $\beta$-actin (cat. no. ab8226) were purchased from Abcam (Cambridge, UK). Antibodies against tumor necrosis factor (TNF)- $\alpha$ (cat. no. 11948P) and Notch 1 (cat. no. 4380P) were purchased from Cell Signaling Technology, Inc. (Danvers, MA, USA). Antibodies against allophycocyanin (APC)-cluster of differentiation (CD)197 (cat. no. 120107), fluorescein isothiocyanate (FITC)-F4/80 (cat. no. 123107), and phycoerythrin (PE)-CD206 (cat. no. 141705) were purchased from BioLegend, Inc. (San Diego, CA, USA). Horseradish-peroxidase conjugated secondary antibodies for western blot (WB) analysis were purchased from Abcam (cat. no. ab150157). Recombinant mouse vascular endothelial growth factor (VEGF) was purchased from R\&D Systems Europe, Ltd. (Abingdon, UK).
IPSC-MSCs and Raw 264.7 cells co-culture. Naive IPSC-MSCs and Raw 264.7 cells were cultured in collagen-coated dishes with High Glucose Dulbecco's modified Eagle's medium (DMEM; 4.5 g/l glucose; Gibco; Thermo Fisher Scientific, Inc.) supplemented with $10 \%$ heat-inactivated fetal bovine serum (Gibco; Thermo Fisher Scientific, Inc.) and $1 \%$ penicillin/streptomycin at $37^{\circ} \mathrm{C}$ in a humidified $5 \% \mathrm{CO}_{2}$ atmosphere. Medium was changed every 2-3 days. By 3-4 days of incubation, cells had reached $70-80 \%$ confluence and were seeded into 6-well flat bottom microtitre plates at a cell density of $1 \times 10^{6}$ cells/well. IPSC-MSCs $\left(1 \times 10^{6}\right.$ cells/well) were inoculated onto the upper membrane (Transwell insert) and Raw 264.7 cells $\left(1 \times 10^{6}\right.$ cells/well) were inoculated into the lower chamber of a conventional double cell co-culture system. The cells were divided into three groups, including Raw 264.7 cells ( $1 \times 10^{6}$ cells/well; control group), Raw 264.7 cells (1x10 6 cells/well; OGD group) and IPSC-MSCs $\left(1 \times 10^{6}\right.$ cells/well $)+$ Raw 264.7 cells $\left(1 \times 10^{6}\right.$ cells/well; OGD + IPSC-MSCs group).

$O G D$. OGD was induced by exposing Raw 264.7 cells to a calibrated gas mixture of $2 \% \mathrm{CO}_{2}, 5 \% \mathrm{CO}_{2}$ and $93 \% \mathrm{~N}_{2}$ in a 3 -gas incubator (Forma; Thermo Fisher Scientific, Inc.) in PBS for 0.5, 1, 2 and $4 \mathrm{~h}$. The OGD modeling time was determined by the results of the Cell Counting Kit-8 (CCK-8) assay. Control cells were maintained in normal conditions $\left(5 \% \mathrm{CO}_{2}, 95 \%\right.$ humidified air) in complete high glucose medium (DMEM $4.5 \mathrm{~g} / \mathrm{l}$ ).

CCK-8 assay. The integrity of cellular function was measured using a CCK-8 assay (formazan crystals were solubilized with $0.1 \mathrm{~N} \mathrm{HCl}$ isopropanol). Raw 264.7 cells exposed to OGD were further incubated for $0.5,1,2$ and $4 \mathrm{~h}$ with $10 \mu \mathrm{lCCK}-8$ at $37^{\circ} \mathrm{C}$ in a normoxic chamber. At the end of the incubation period, absorption was detected at $450 \mathrm{~nm}$, with background subtraction at $630 \mathrm{~nm}$, using a microplate reader (Stat Fax-2100; Awareness Technology, Inc., Palm City, FL, USA) (13).

Flow cytometry (FCM). Cells were harvested, washed in PBS and resuspended in binding buffer, and a $0.5 \mathrm{ml}$ aliquot was withdrawn for analysis. Following the addition of annexin V-FITC, APC and PE (BioLegend, Inc.), the sample was incubated for $30 \mathrm{~min}$ in the dark. Stained cells were analyzed using a BD Biosciences (Franklin Lakes, NJ, USA) flow cytometer. A total of $1 \times 10^{6}$ cells were counted per sample, and the data were processed using Beckman Coulter Cell Lab Quanta ${ }^{\mathrm{TM}} \mathrm{SC}$ MPL (AL510171; Beckman Coulter, Inc., Brea, CA, USA).

The CCK-8 and the cell sorting staining assays for FCM were performed in parallel in twin cultures that were subjected to identical conditions. This procedure was adopted to eliminate variations in the cell population, growth conditions and experimental procedures.

WB analysis. 10X RIPA lysis buffer (Abcam) was used for protein extraction (cat. no. ab156034) and a BCA protein assay kit was used for protein determination. A total of $20 \mu \mathrm{g}$ denatured protein diluted in $20 \mu \mathrm{l}$ solution samples were loaded on a $10 \%$ SDS-PAGE gel, and electrophoresis was run at $150 \mathrm{~V}$ for $1 \mathrm{~h}$. Proteins were transferred to a polyvinylidene fluoride membrane (BioRad Laboratories, Inc., Hercules, CA, USA) using a Trans-Blot semi-dry transfer. Cell membranes were blocked with $5 \%$ skimmed milk in TBS-Tween 20 at $4^{\circ} \mathrm{C}$ 
Table I. Reverse transcription-quantitative polymerase chain reaction primers.

Primer sequence (5'-3')

\begin{tabular}{|c|c|c|c|c|}
\hline Symbol & Gene ID & Amplicon size, bp & Forward & Reverse \\
\hline Nos2 (iNOS) & 18126 & 127 & GTTCTCAGCCCAACAATACAAGA & GTGGACGGGTCGATGTCAC \\
\hline Abl2 (Arg1) & 11352 & 198 & GAGCCACCGTTTTACATTGTGA & CTCGCCCACTAGGCAGTTC \\
\hline Notch1 & 18128 & 227 & ACACCGTGTAAGAATGCTGGA & GCCTGCTGACATGATTTTCCTG \\
\hline Atcay (Hes1) & 16467 & 157 & TCCGACGACTTCCTCGACA & CACCAGGCATGTTTTTGGCG \\
\hline$\beta$-actin & 11461 & 154 & GGCTGTATTCCCCTCCATCG & CCAGTTGGTAACAATGCCATGT \\
\hline
\end{tabular}

iNOS, inducible nitric oxide synthase; Arg1, arginase 1; Notch1, neurogenic locus notch homolog protein 1; Hes1, transcription factor HES-1.

for $1 \mathrm{~h}$ and subsequently incubated with primary antibodies against TNF- $\alpha$, iNOS, Notch1, Hes1, IL-10, Arg1 and $\beta$-actin at a dilution of 1:1,000 for $1 \mathrm{~h}$ at room temperature. Following incubation with the primary antibodies, the secondary antibody $(1: 1,000)$ was used and membranes were incubated at room temperature for $1 \mathrm{~h}$. The membranes were treated with an enhanced chemiluminescence substrate (Thermo Fisher Scientific, Inc.) for 1-2 min and exposed at different exposure times. $\beta$-actin $(1: 5,000)$ was used as the loading control. Blots from 4-6 different experiments were scanned and band intensities from each blot were analyzed using Image J software (version 1.8.0_101; National Institutes of Health, Bethesda, MD, USA) and expressed relative to the $\beta$-actin signal.

$R N A$ extraction and reverse transcription-quantitative polymerase chain reaction ( $R T-q P C R)$ analysis. Total RNA was isolated from cells using TRIzol reagent (Takara Bio, Inc., Otsu, Japan) according to the manufacturer's protocol. For RT, $1 \mu \mathrm{g}$ total RNA from each sample was reverse transcribed using Superscript II Reverse Transcriptase (Takara Bio, Inc.). Aliquots of diluted cDNA (1:5) were amplified using TransStart Top Green qPCR SuperMix in a final volume of $20 \mu \mathrm{l}$. The RT reaction was performed at $30^{\circ} \mathrm{C}$ for $10 \mathrm{~min}$, $42^{\circ} \mathrm{C}$ for $60 \mathrm{~min}$, and $70^{\circ} \mathrm{C}$ for $10 \mathrm{~min}$. qPCR amplification was performed using a LabCycler Real-Time PCR system (SensoTech GmbH, Magdeburg-Barleben, Germany) using SYBR Green dye (Takara Bio, Inc.), and protein quantification was performed using the $2^{-\Delta \Delta C q}$ method (14). The sequences of the primers are listed in Table I.

Data and statistical analysis. The values are presented as the mean \pm standard deviation. The control, OGD and OGD + IPSC-MSC groups were tested for normality within all the time points using repeated measures analysis of variance followed by the Tukey post hoc test. The null hypothesis was rejected at the significance level $\alpha<0.05$. Data were analyzed statistically using SPSS 22.0 software (IBM Corp., Armonk, $\mathrm{NY}$, USA). $\mathrm{P}<0.05$ was considered to indicate a statistically significant difference.

\section{Results}

Establishment OGD model and CCK-8 assay. Co-cultured Raw 264.7 and IPSC-MSCs are presented in Fig. 1. Following establishment of the OGD model, cell viability was determined by assessing the integrity of mitochondrial function using the CCK-8 assay. The CCK-8 assay demonstrated that the cell viability of the OGD group and the OGD + IPSC-MSC group decreased during 0-4 h. The OGD $(0.59+0.02)$ and OGD + IPSC-MSC groups $(0.61+0.01)$ exhibited no apparent alterations in cell viability at $0.5 \mathrm{~h}$ compared with the control group ( $\mathrm{P}>0.05$ ); however, cell viability decreased following $1-2 \mathrm{~h}$ of intervention. In addition, OGD cells and OGD + IPSC-MSC cell viability decreased more significantly following intervention for $4 \mathrm{~h}$. The cell viability of the OGD group $(0.38 \pm 0.13)$ and the OGD + IPSC-MSC group $(0.42 \pm 0.10)$ exhibited a statistically significant decrease compared with the control group (0.78 \pm 0.05$)(\mathrm{P}=0.032, \mathrm{P}<0.05$; Fig. 2).

Effect of OGD and IPSC-MSC intervention on Raw 264.7 macrophage polarization. To determine whether OGD and IPSC-MSC intervention affected macrophage polarization, FCM, WB and RT-qPCR analyses were performed. FCM analysis was used to objectively analyze the proportion of M1 and M2 macrophages. Following quantitative analysis, it was observed that the proportion of M1 and M2 macrophages following intervention for $4 \mathrm{~h}$ was statistically significant among the control group (M1, 27.59 $\pm 1.3 \%$; M2, 52.59 $\pm 11.0 \%$ ), OGD + IPSC-MSC group (M1, 50.02 $\pm 2.4 \%$; M2, 37.08 $\pm 10.4 \%$ ), and OGD group (M1, $56.97 \pm 12.8 \%$; M2, 33.96 $\pm 9.7 \%$; $\mathrm{P}<0.05)$. The proportion of $\mathrm{M} 1$ and M2 macrophages between the OGD group (M1, 83.55 $\pm 7.3 \%$; $\mathrm{M} 2,11.41 \pm 3.2 \%$ ) and control group (M1, 29.34 $4.1 \%$; M2, $52.34 \pm 5.4 \%$ ) was also statistically significant at $6,12,24$ and $48 \mathrm{~h}$ $(\mathrm{P}=0.026 ; \mathrm{P}<0.05)$, and over time the trend was more apparent. The proportion of M1 and M2 macrophages between the OGD

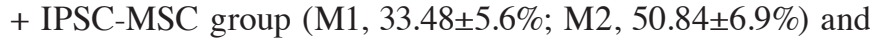
OGD group (M1, 83.55 $\pm 7.3 \%$; M2, $11.41 \pm 3.2 \%$ ) was statistically significant at 4, 6, 12, 24 and $48 \mathrm{~h}(\mathrm{P}=0.037, \mathrm{P}<0.05)$, and over time the trend was more apparent (Fig. 3). To confirm the FCM results, WB and RT-qPCR analyses were performed. WB and RT-qPCR analysis indicated a decrease in Arg1 and IL-10 in the OGD group compared with the control group at 6, 12, 24 and $48 \mathrm{~h}$ $(\mathrm{P}=0.041, \mathrm{P}<0.05$; Figs. 4 and $5 \mathrm{~A}-\mathrm{C})$. In addition, a significant decrease in iNOS and TNF- $\alpha$ levels in the OGD + IPSC-MSC group was observed at $6,12,24$ and $48 \mathrm{~h}$ compared with the OGD group $(\mathrm{P}=0.018, \mathrm{P}<0.05)$, and significantly increased at 24 and 48 h compared with the control group $(\mathrm{P}=0.026, \mathrm{P}<0.05$; Figs. 4 and 5A, D and E). 
RAW 264.7

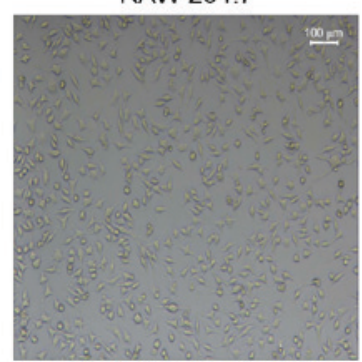

Co-incubate day 1

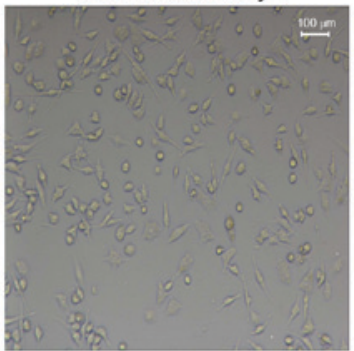

IPSCs-MSCs

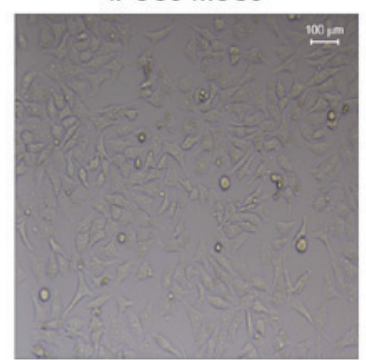

Co-incubate day 2

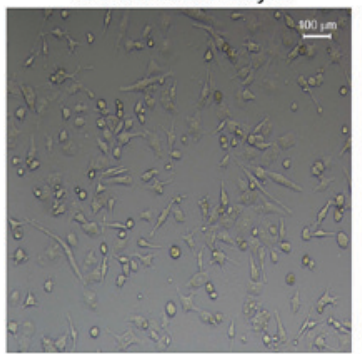

Figure 1. Cells were cultured under normal conditions (control). IPSC-MSCs and Raw 264.7 cells were co-incubated under normal conditions on days 1 and 2. Representative images from at least three independent experiments are presented (magnification, x100). IPSC-MSCs, induced pluripotent stem cell-derived mesenchymal stem cells.

Effects of IPSC-MSC intervention on the polarization of Raw 264.7 macrophages through the Notch-1 pathway. The Notch gene encodes a highly conserved cell surface receptor that regulates the development of a variety of biological cells, ranging from sea urchins to humans. Therefore, the present study aimed to examine the expression of Notch-1 in the OGD + IPSC-MSC group. Notch-1 protein and mRNA levels were determined in each group. WB quantitative analysis demonstrated that Notch-1 expression in the OGD + IPSC-MSC group [0.60 \pm 0.05 Absorbance Unit (A.U.)] was significantly decreased at 6,12,24 and $48 \mathrm{~h}$ compared with the OGD group (1.12 \pm 0.11 A.U.), and significantly increased at 24 and 48 time intervals compared with the control group $(0.86 \pm 0.07$ A.U.) ( $\mathrm{P}=0.034, \mathrm{P}<0.05$; Fig. 5A and F). Additionally, RT-qPCR analysis confirmed the effect of IPSC-MSC intervention on the polarization of Raw 264.7 macrophages through the Notch-1 pathway. A significant increase in Notch-1 in the OGD group was observed compared with the control group at 6, 12, 24 and 48 h (OGD, 3.44 \pm 0.22 A.U.; $\mathrm{P}=0.014$, $\mathrm{P}<0.05$; Fig. 4).

Effects of IPSC-MSC intervention on Hesl and the polarization of Raw 264.7 macrophages post-OGD. In order to further characterize the effects of Notch-1 in the OGD model, Hes1, a downstream effector of Notch-1, was examined by WB and RT-qPCR analyses. Densitometric analysis of Hes1 WB bands demonstrated a significant decrease in Hes1 expression in the OGD + IPSC-MSC group (1.69 \pm 0.17 A.U.) at 6, 12, 24 and 48 h compared with the OGD group (2.01 \pm 0.07 A.U.); however, Hes1 expression in the OGD + IPSC-MSC group was significantly increased compared with control groups at 6,12 , 24 and $48 \mathrm{~h}$ time intervals $(1.58 \pm 0.21$ A.U.; $\mathrm{P}=0.036, \mathrm{P}<0.05$; Fig. 5A and G). Quantitative analysis of the PCR results demonstrated a significant decrease in Hes1 expression in the OGD + IPSC-MSC group (2.5 \pm 0.37 A.U.) compared with the

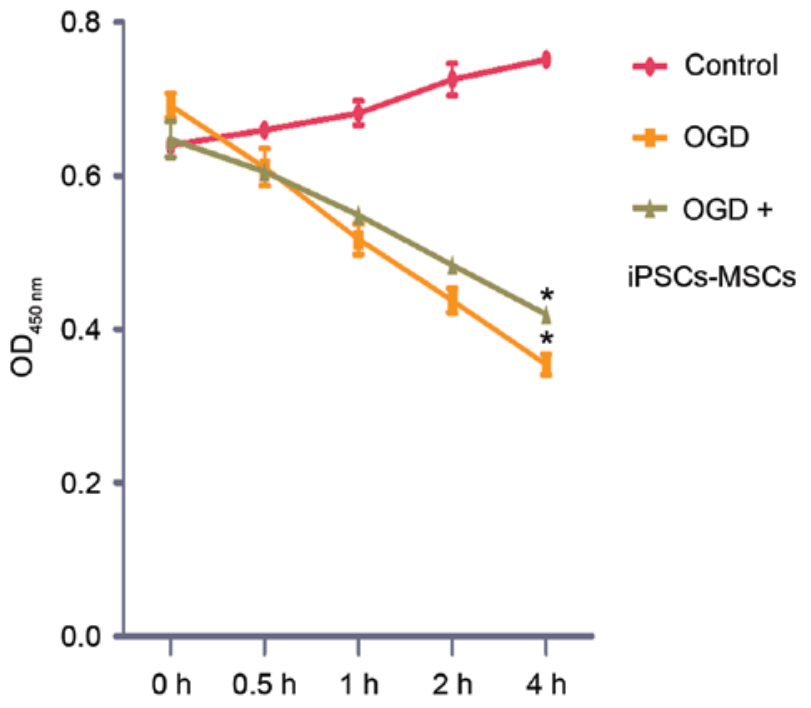

Figure 2. Control cells were incubated in a normoxic chamber. OGD cells and OGD + IPSC-MSCs were exposed to OGD $(n=3)$. The cell viability of the OGD group and the OGD + IPSC-MSC group exhibited a statistically significant decrease compared with the control group $(\mathrm{P}<0.05)$. ${ }^{*} \mathrm{P}<0.05$ vs. control group. OGD, oxygen and glucose deprivation; OD, optical density.

OGD group ( $3.50 \pm 0.27$ A.U), and a significant increase in Hes1 expression in the OGD + IPSC-MSC group compared with the control group (1.00 A.U.; $\mathrm{P}=0.031, \mathrm{P}<0.05$; Fig. 4).

\section{Discussion}

Cerebral hypoxic ischemia injury is a primary cause of mortality and disability in emergency medicine (15). Although improvements in CPR performance and the increasing success rate in achieving recovery of spontaneous respiration and circulation in recent years, the survival and discharge rate of patients post-sudden cardiac arrest remain poor (16). However, in Denmark and the USA, greater survival and favorable neurological status were associated with hospital based post-resuscitative care guidelines; therefore, study of the associated mechanisms is required $(17,18)$. Macrophages, a particular type of immune cell, serve a role in innate immunity, including killing pathogens. A previous study demonstrated the role of macrophage polarization direction in anti-tumor immunity, infection, immune responses, atherosclerosis, cardiovascular disease and diabetes (19). In addition, glucose tolerance abnormalities and other diseases have served an important role (19). A study demonstrated that axonal regeneration may be the primary target of and key concern with nerve injury repair (20). Previous studies aiming to promote axonal regeneration have demonstrated that the primary reason for the failure of early regeneration in the adult mammalian central nervous system (CNS) is the existence of myelin inhibitors $(21,22)$. Studies have confirmed that macrophages/glial cells in the CNS exhibit chemotaxis, phagocytosis of myelin fragments, and that it is possible to improve the prognosis of neurological function $(23,24)$. However, previous studies have demonstrated that macrophages mediate inflammatory responses in the process of neuronal repair, resulting in secondary neuronal damage $(25,26)$. Therefore, in-depth examination of the regulation of macrophages in the CNS 
A

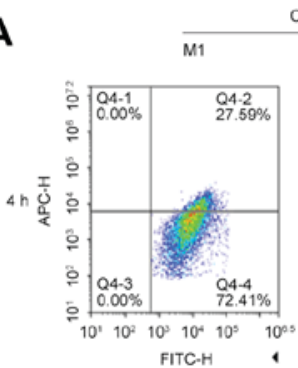

Control
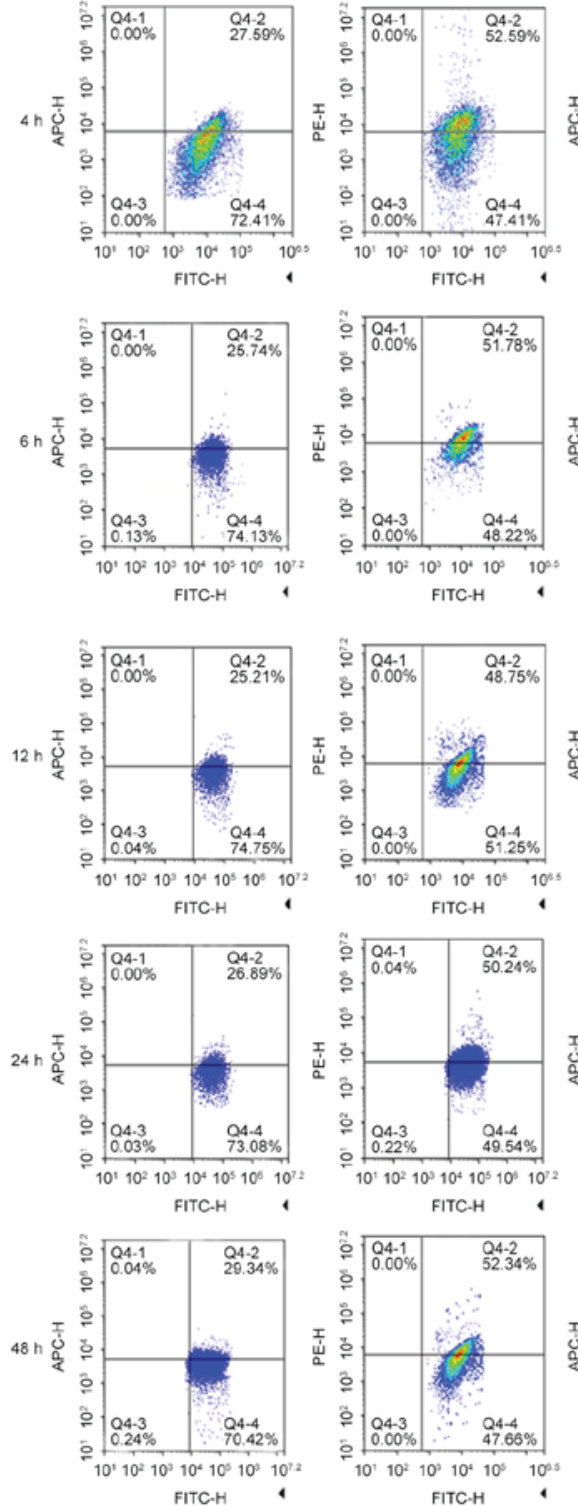

\begin{tabular}{ll} 
OGD \\
\hline M1
\end{tabular}
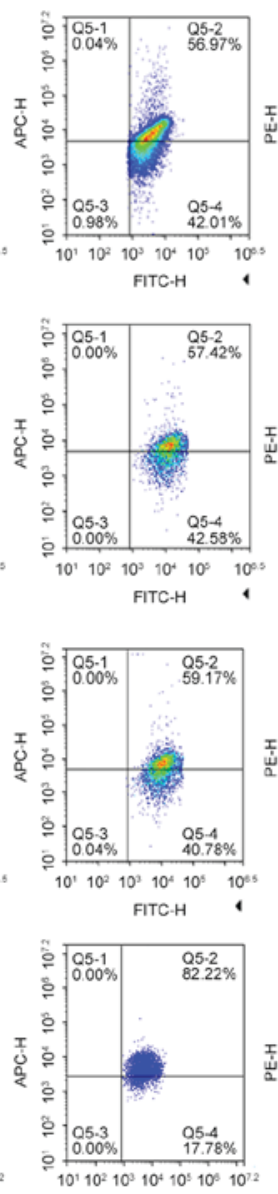

FITC-H

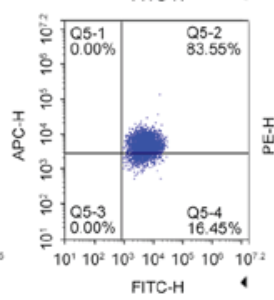

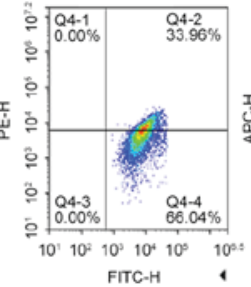
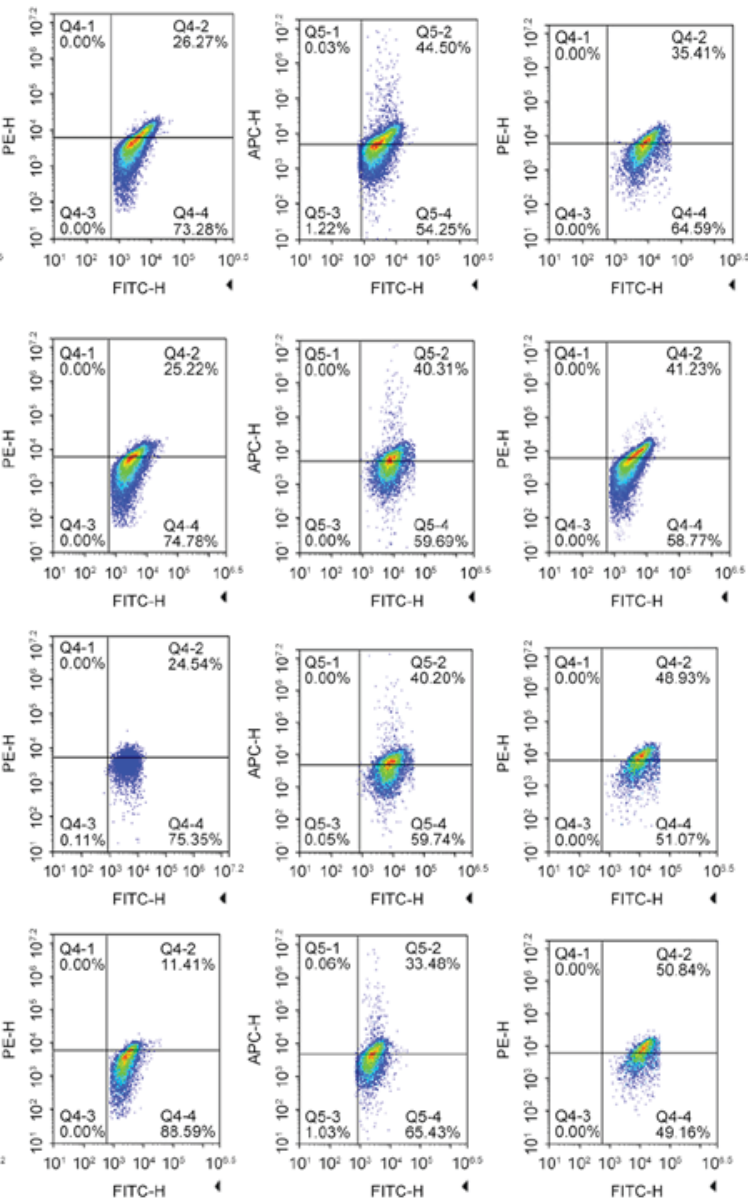
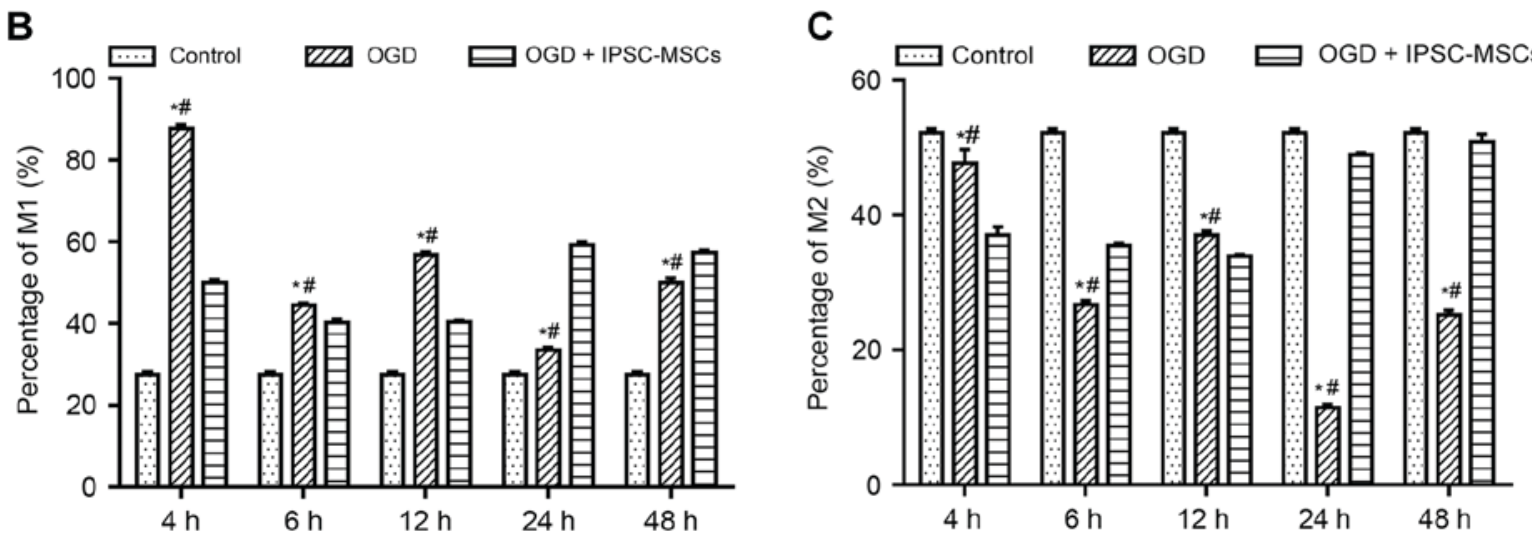

Figure 3. (A) Flow cytometry analysis of the control, OGD and OGD + IPSC-MSC groups at the 4, 6, 12, 24 and $48 \mathrm{~h}$ time points (n=3). The bar graphs illustrate the (B) M1 and (C) M2 macrophage proportions in the control, OGD and OGD + IPSC-MSC groups at the 4, 6, 12, 24 and 48 h time intervals (n=3). ${ }^{*} \mathrm{P}<0.05$ vs. control group; ${ }^{\text {P }}<0.05$ vs. OGD + IPSC-MSC group. OGD, oxygen and glucose deprivation; IPSC-MSCs, induced pluripotent stem cell-derived mesenchymal stem cells.

may improve the prognosis of neurological function following cardiac arrest. In the present study, the results demonstrated that intervention with IPSC-MSCs affected the polarization of Raw 264.7 macrophages via the Notch-1/Hes1 signaling 


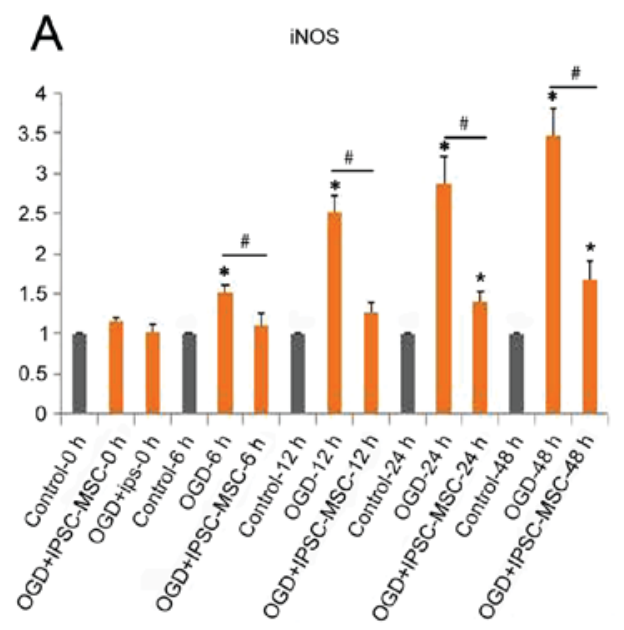

B Arg1
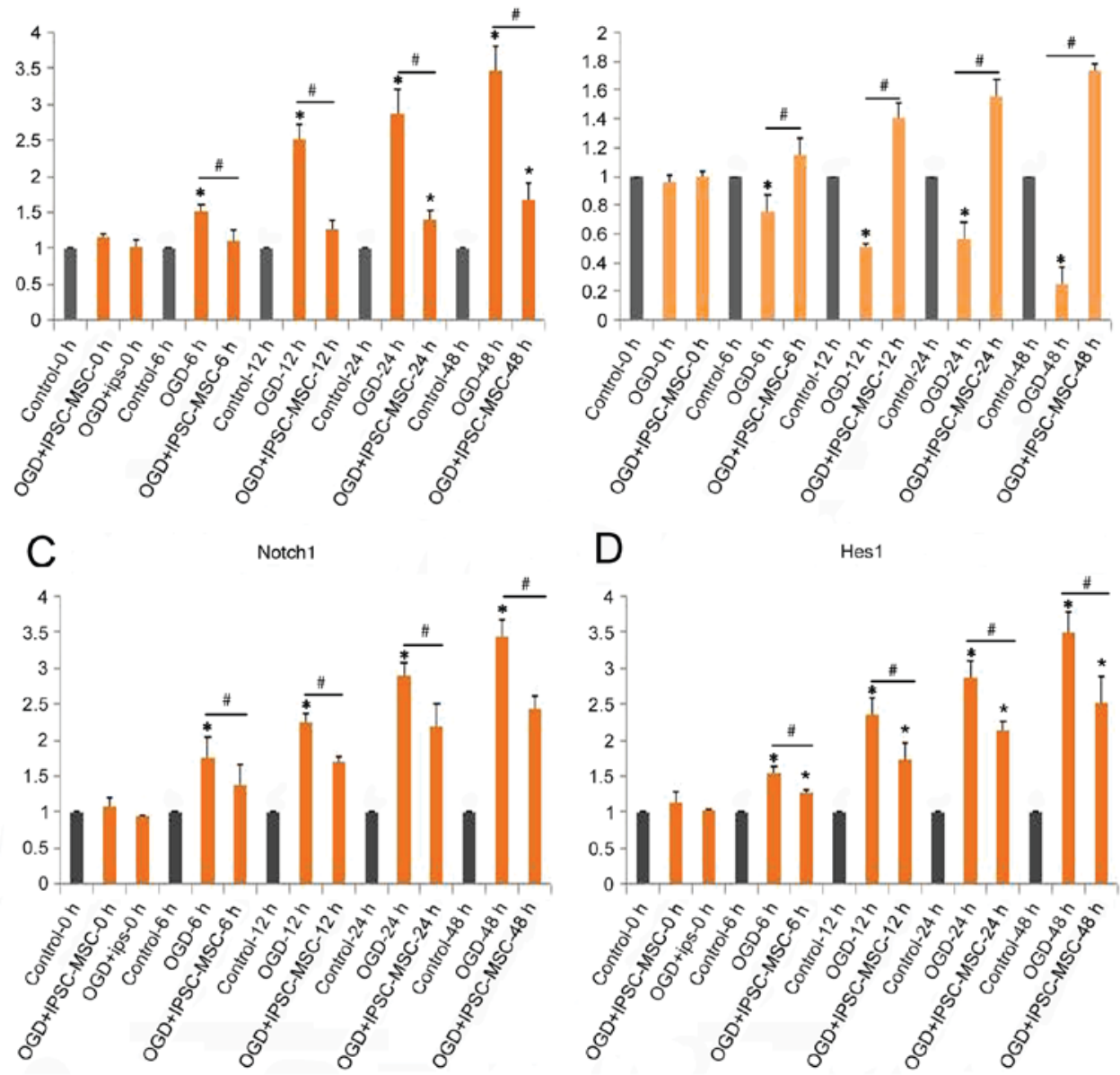

D Hes 1

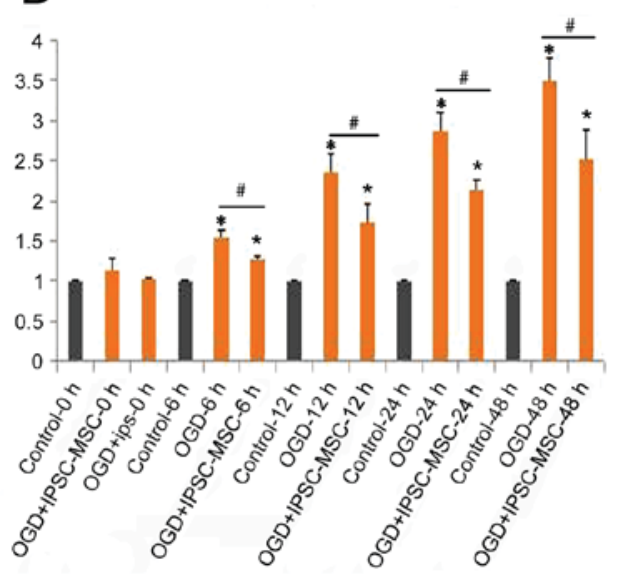

Figure 4. Reverse transcription-quantitative polymerase chain reaction analysis of the control, OGD and OGD + IPSC-MSC groups regarding the expression levels of (A) iNOS, (B) Arg1, (C) Notch1 and (D) Hes1. Each bar represents the mean \pm standard error of the mean ( $\mathrm{n}=3$ ). ${ }^{*} \mathrm{P}<0.05$ vs. control group; ${ }^{*} \mathrm{P}<0.05$ vs. OGD + IPSC-MSC group. OGD, oxygen and glucose deprivation; IPSC-MSCs, induced pluripotent stem cell-derived mesenchymal stem cells; iNOS, inducible nitric oxide synthase; Arg1, arginase 1; Notch1, neurogenic locus notch homolog protein 1; Hes1, transcription factor HES-1.

pathway. However, additional experiments are required to support the present results.

In previous studies, IPSC-MSCs have been induced by an alteration in macrophage polarization direction to achieve a protective effect in inflammatory diseases (27-29). M2 macrophages serve an important role in combatting excessive inflammatory injury and tissue repair during pathogen infection (30). Following an injection of IPSC-MSCs, the expression of $\mathrm{CD}_{206}{ }^{+}$(M2) macrophages was observed to increase in acute kidney-injured mice (31). IPSC-MSCs are able to reduce renal tubular injury, reduce interstitial fibrosis, and increase the CD206 ${ }^{+} \mathrm{CD} 206^{-}$proportion of macrophages in patients with unilateral fallopian tube obstruction caused by aseptic nephritis (32). IPSC-MSC intervention in a myocardial infarction model was confirmed to alter the direction of M2 macrophage polarization (33). In addition, IPSC-MSCs exert a protective effect on the phenotype and function of macrophages during severe infections. Krasnodembskaya et al (34) demonstrated that an injection of IPSC-MSCs may increase the proportion of CD206 macrophages in the spleens of mice. A previous study demonstrated that mice lacking the Notch-recombining binding protein suppressor of hairless pathway in macrophages produce lower levels of specific types of M1 macrophage and, therefore, exhibit a low-inflammation phenotype (35). These results indicated that IPSC-MSCs may regulate the surface molecules of macrophages to increase the levels of $\mathrm{CD}_{206}{ }^{+}$macrophages, accompanied by the role of tissue repair and other protective effects (36-39). Therefore, it was hypothesized that IPSC-MSCs may alter the polarization of macrophages, which may be a central link in the immune regulatory network of brain resuscitation and improve outcomes following cardiac arrest.

Arg1, IL-10, iNOS and TNF- $\alpha$ expression levels confirmed that IPSC-MSCs may regulate the balance between inflammation and anti-inflammation, reduce the inflammatory responses of the body, inhibit inflammatory reactions, and induce phenotypic alterations and functions of macrophages $(40,41)$. However, the mechanisms of how IPSC-MSCs alter the polarization of macrophages remain unclear.

Previous studies have determined that IPSC-MSC signaling pathways are involved in the differentiation of macrophages, including the c-Jun $\mathrm{N}$-terminal kinase, phosphatidylinositol 3-kinase/RAC- $\alpha$ serine/threonine-protein kinase, Notch and tyrosine-protein kinase JAK/signal transducer and activator of transcription signaling pathways $(42,43)$. 
A
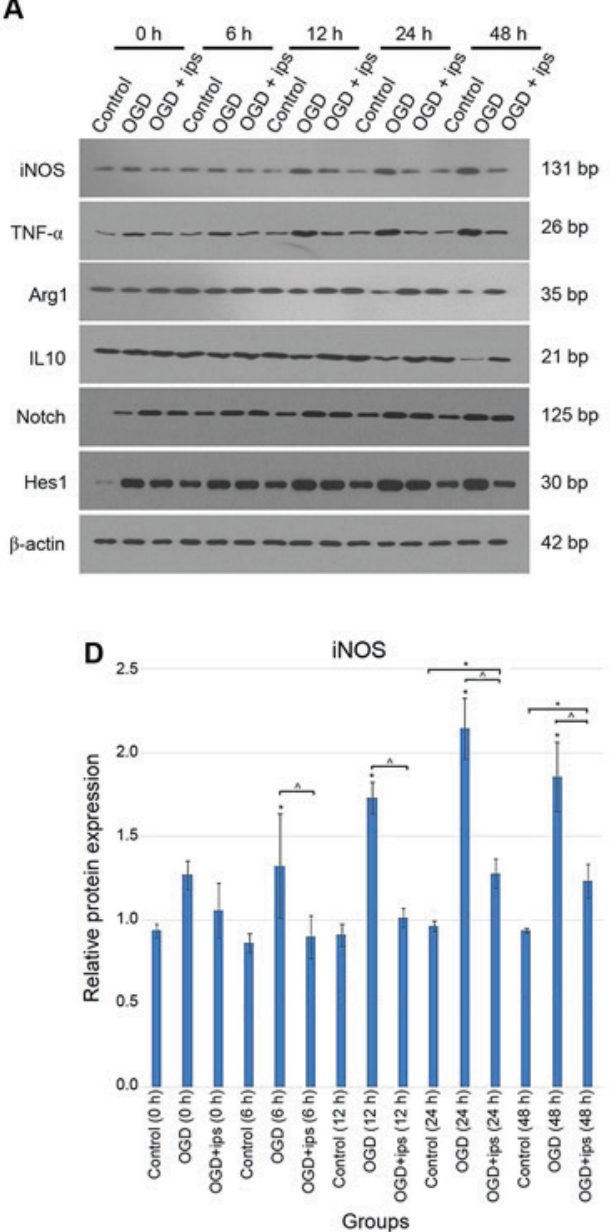

G

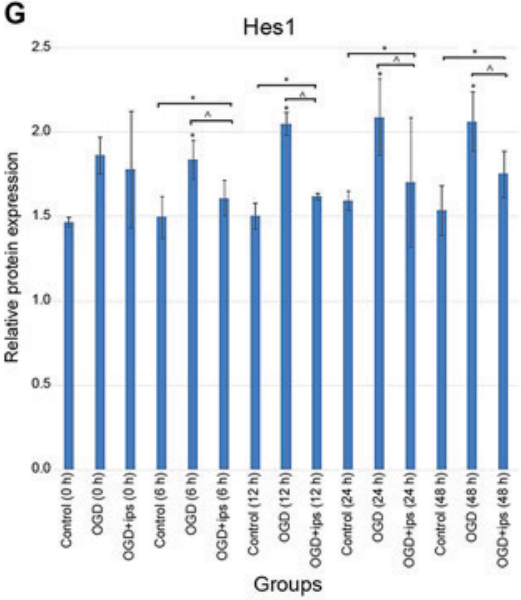

B

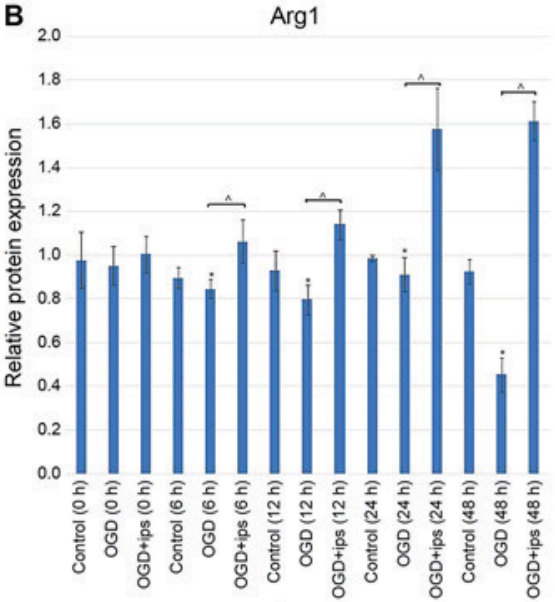

$\mathbf{E}_{2.5}$

Groups

TNF- $\alpha$

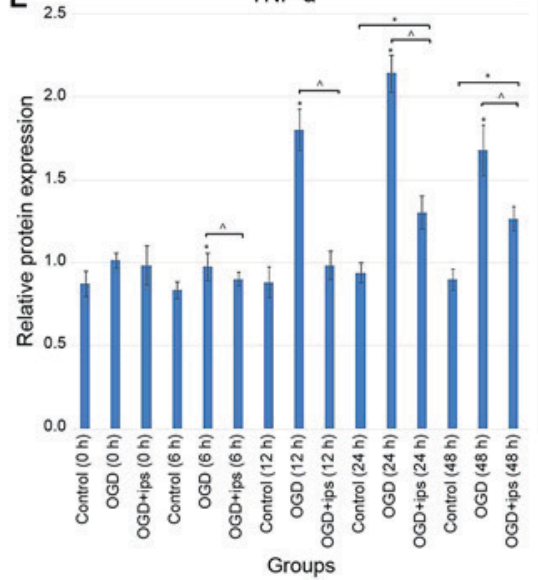

H

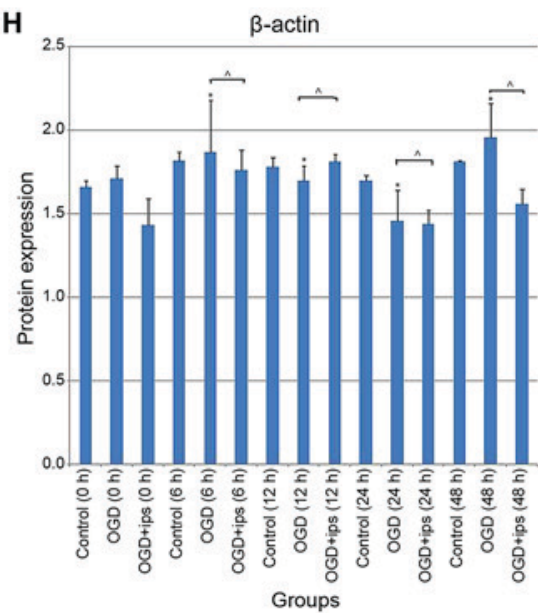

C

IL-10
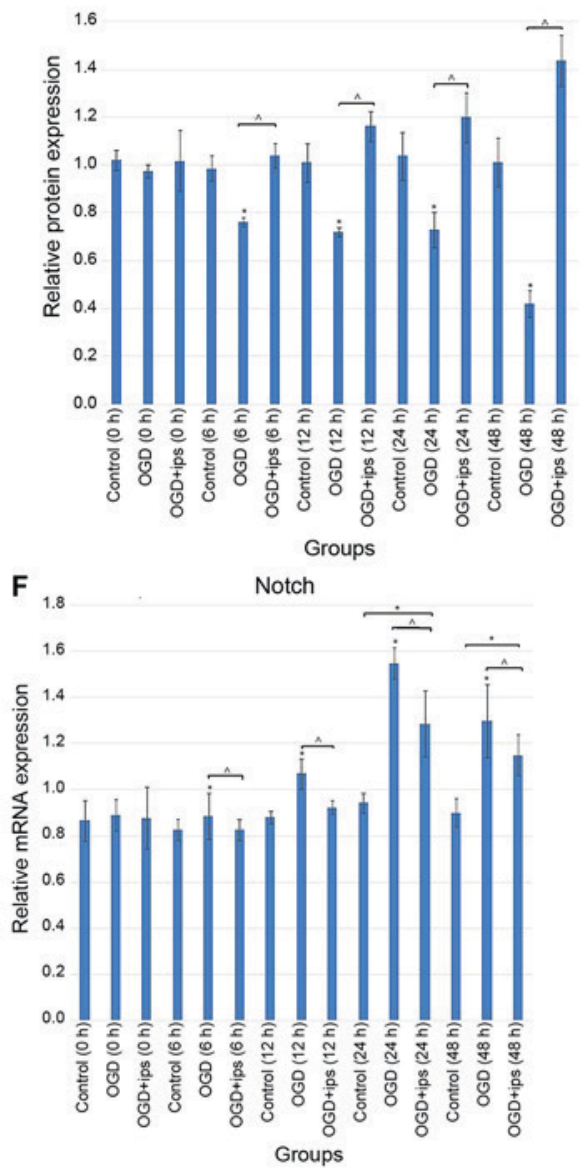

Figure 5. (A) Representative western blot analysis of the control, OGD and OGD + IPSC-MSC groups at the 0,6, 12, 24 and $48 \mathrm{~h}$ time points. Quantification of the expression of (B) Arg1, (C) IL-10, (D) iNOS, (E) TNF- $\alpha$ (F) Notch-1 (G) Hes1 and (H) $\beta$-actin at 6, 12, 24 and 48 h time intervals for the corresponding control, OGD and OGD + IPSC-MSC groups. Each bar represents the mean \pm standard error of the mean ( $\mathrm{n}=3)$. ${ }^{*} \mathrm{P}<0.05$ vs. control; ${ }^{\wedge} \mathrm{P}<0.05$ vs. OGD + IPSC-MSC group. IL-10, interleukin 10; Notch-1, neurogenic locus notch homolog protein-1; Hes1, transcription factor HES-1; iNOS, inducible nitric oxide synthase; TNF- $\alpha$, tumor necrosis factor- $\alpha$; Arg1, arginase 1; OGD, oxygen and glucose deprivation; IPSC-MSCs, induced pluripotent stem cell-derived mesenchymal stem cells; OGD + ips, OGD + IPSC-MSC group.

The Notch gene encodes a highly conserved cell surface receptor that regulates the development of a variety of biological cells, ranging from sea urchins to humans. The Notch signaling pathway consists of the Notch receptor, delta-serrate-LAG-2 protein (Notch ligand), CSL protein, DNA binding proteins and other regulatory molecules. The Notch-1 signaling pathway is directly associated with inflammation and anti-inflammatory reactions. Upregulation of the Notch-1 signaling pathway enhances the ability of macrophages to kill pathogens (44). During the activation of the Notch-1 signaling pathway, TNF-a and IL-6 expression levels increase, inhibiting IL-6, which causes Notch-1 levels to decrease and leads to a reduced inflammatory response. Therefore, Notch-1 may be an important signaling pathway 
in the regulation of macrophage polarization and functional status (45). IPSC-MSC intervention may decrease the apoptosis of macrophages in the OGD model $(46,47)$. The use of a Notch-1 receptor blocking agent or the disruption of a downstream signaling pathway may lead to a decrease in the expression of pro-inflammatory factors and an increase in the expression of anti-inflammatory factors, which cause M2 macrophages to bypass activation. The strength of the present study is that it demonstrated that intervention with IPSC-MSCs affected the polarization of Raw 264.7 macrophages via the Notch-1/Hes-1 pathway. However, there were limitations to the present study. A Notch inhibitor was not used and only macrophage polarization was observed. A grouping experiment for the dose of IPSCs-MSCs was not performed. Other channel-associated factors were not detected and, in future, in vivo experiments are required for functional verification.

In the present study, WB and RT-qPCR analyses confirmed that IPSC-MSC intervention affected the polarization of Raw 264.7 macrophages via the Notch-1 pathway. RT-qPCR analysis demonstrated a significant decrease in Hesl expression in the OGD + IPSC-MSCs group compared with the OGD group; and demonstrated a significant increase compared with the control group. Therefore, the results of the present study demonstrated that intervention with IPSC-MSCs may influence the polarization of Raw 264.7 macrophages via the Notch-1/Hes1 signaling pathway.

\section{Acknowledgements}

Not applicable.

\section{Funding}

The present study was supported by grants from the National Natural Science Foundation of China (grant no. NSFC-2013-81372022), Science and Technology Planning Project of Guangdong Province, China (grant no. 20140221), the Department of Cardiology, Heart Center, The First Affiliated Hospital, Sun Yat-Sen University, and the Key Laboratory on Assisted Circulation, Ministry of Health (Guangzhou, China).

\section{Availability of data and materials}

The datasets used or analyzed during the current study are available from the corresponding author on reasonable request.

\section{Authors' contributions}

XJ made substantial contributions to the conception and design of the present study. YY and DW interpreted the data. HL and YL gave final approval to the manuscript to be published and co-cultured the IPSC-MSCs and Raw 264.7 cells. ZX and JW performed RT-qPCR, western blot analysis, FCM and the CCK-8 assay.

\section{Ethics approval and consent to participate}

Not applicable.

\section{Consent for publication}

Not applicable.

\section{Competing interests}

The authors declare that they have no competing interests.

\section{References}

1. Benjamin EJ, Blaha MJ, Chiuve SE, Cushman M, Das SR, Deo R, de Ferranti SD, Floyd J, Fornage M, Gillespie C, et al: Heart disease and stroke statistics-2017 update: A report from the American heart association. Circulation 135: e146-e603, 2017.

2. Adrie C, Adib-Conquy M, Laurent I, Monchi M, Vinsonneau C, Fitting C, Fraisse F, Dinh-Xuan AT, Carli P, Spaulding C, et al: Successful cardiopulmonary resuscitation after cardiac arrest as a 'sepsis-like' syndrome. Circulation 106: 562-568, 2002.

3. Lian Q, Zhang Y, Zhang J, Zhang HK, Wu X, Zhang Y, Lam FF, Kang S, Xia JC, Lai WH, et al: Functional mesenchymal stem cells derived from human induced pluripotent stem cells attenuate limb ischemia in mice. Circulation 121: 1113-1123, 2010.

4. Zheng Y, Cai W, Zhou S, Xu L and Jiang C: Protective effect of bone marrow derived mesenchymal stem cells in lipopolysaccharide-induced acute lung injury mediated by claudin- 4 in a rat model. Am J Translat Res 8: 3769-3779, 2016.

5. Li Y, Xu J, Shi W, Chen C, Shao Y, Zhu L, Lu W and Han X: Mesenchymal stromal cell treatment prevents H9N2 avian influenza virus-induced acute lung injury in mice. Stem Cell Res Ther 7: 159, 2016.

6. Liang ZX, Sun JP, Wang P, Tian Q, Yang Z and Chen LA: Bone marrow-derived mesenchymal stem cells protect rats from endotoxin-induced acute lung injury. Chin Med J (Engl) 124: 2715-2722, 2011.

7. Lai TS, Wang ZH and Cai SX: Mesenchymal stem cell attenuates neutrophil-predominant inflammation and acute lung injury in an in vivo rat model of ventilator-induced lung injury. Chin Med J (Engl) 128: 361-367, 2015.

8. Gupta N, Su X, Popov B, Lee JW, Serikov V and Matthay MA: Intrapulmonary delivery of bone marrow-derived mesenchymal stem cells improves survival and attenuates endotoxin-induced acute lung injury in mice. J Immunol 179: 1855-1863, 2007.

9. Li Y, Zhao T, Liu B, Halaweish I, Mazitschek R, Duan X and Alam HB: Inhibition of histone deacetylase 6 improves long-term survival in a lethal septic model. J Trauma Acute Care Surg 78: 378-385, 2015.

10. Kikuchi S, Nishihara T, Kawasaki S, Abe N, Kuwabara J, Choudhury ME, Takahashi H, Yano H, Nagaro T, Watanabe Y, etal: The ameliorative effects of a hypnotic bromvalerylurea in sepsis. Biochem Biophys Res Commun 459: 319-326, 2015.

11. Sun L, Sun G, Yu Y and Coy DH: Is Notch signaling a specific target in hepatocellular carcinoma? Anticancer Agents Med Chem 15: 809-815, 2015.

12. Tachikawa Y, Matsushima T, Abe Y, Sakano S, Yamamoto M, Nishimura J, Nawata H, Takayanagi R and Muta K: Pivotal role of Notch signaling in regulation of erythroid maturation and proliferation. Eur J Haematol 77: 273-281, 2006.

13. Kritis A, Pourzitaki C, Klagas I, Chourdakis M and Albani M: Proteases inhibition assessment on PC12 and NGF treated cells after oxygen and glucose deprivation reveals a distinct role for aspartyl proteases. PLoS One 6: e25950, 2011.

14. Livak KJ and Schmittgen TD: Analysis of relative gene expression data using real-time quantitative PCR and the 2(-Delta Delta C(T)) method. Methods 25: 402-408, 2001.

15. Lai JCY, Rocha-Ferreira E, Ek CJ, Wang X, Hagberg H and Mallard C: Immune responses in perinatal brain injury. Brain Behav Immun 63: 210-223, 2017.

16. Chokengarmwong N, Ortiz LA, Raja A, Goldstein JN, Huang F and Yeh DD: Outcome of patients receiving CPR in the ED of an urban academic hospital. Am J Emerg Med 34: 1595-1599, 2016.

17. Stub D, Schmicker RH, Anderson ML, Callaway CW, Daya MR, Sayre MR, Elmer J, Grunau BE, Aufderheide TP, Lin S, et al: Association between hospital post-resuscitative performance and clinical outcomes after out-of-hospital cardiac arrest. Resuscitation 92: 45-52, 2015. 
18. Wissenberg M, Lippert FK, Folke F, Weeke P, Hansen CM, Christensen EF, Jans H, Hansen PA, Lang-Jensen T, Olesen JB, et al: Association of national initiatives to improve cardiac arrest management with rates of bystander intervention and patient survival after out-of-hospital cardiac arrest. JAMA 310: 1377-1384, 2013.

19. Zanganeh S, Hutter G, Spitler R, Lenkov O, Mahmoudi M, Shaw A, Pajarinen JS, Nejadnik H, Goodman S, Moseley M, et al: Iron oxide nanoparticles inhibit tumour growth by inducing pro-inflammatory macrophage polarization in tumour tissues. Nat Nanotechnol 11: 986-994, 2016

20. Woolf CJ and Bloechlinger S: Neuroscience. It takes more than two to Nogo. Science 297: 1132-1134, 2002.

21. Ntranos A and Casaccia P: Bromodomains: Translating the words of lysine acetylation into myelin injury and repair. Neurosci Lett 625: 4-10, 2016.

22. Liu Y, Kelamangalath L, Kim H, Han SB, Tang X, Zhai J, Hong JW, Lin S, Son YJ and Smith GM: NT-3 promotes proprioceptive axon regeneration when combined with activation of the mTor intrinsic growth pathway but not with reduction of myelin extrinsic inhibitors. Exp Neurol 283: 73-84, 2016.

23. Zhang K, Zheng J, Bian G, Liu L, Xue Q, Liu F, Yu C, Zhang H, Song B, Chung SK, et al: Polarized macrophages have distinct roles in the differentiation and migration of embryonic spinal-cord-derived neural stem cells after Grafting to injured sites of spinal cord. Mol Ther 23: 1077-1091, 2015.

24. Gruber RC, Ray AK, Johndrow CT, Guzik H, Burek D, de Frutos PG and Shafit-Zagardo B: Targeted GAS6 delivery to the CNS protects axons from damage during experimental autoimmune encephalomyelitis. J Neurosci 34: 16320-16335, 2014.

25. Xiong XY, Liu L and Yang QW: Functions and mechanisms of microglia/macrophages in neuroinflammation and neurogenesis after stroke. Prog Neurobiol 142: 23-44, 2016.

26. Rawji KS, Mishra MK, Michaels NJ, Rivest S, Stys PK and Yong VW: Immunosenescence of microglia and macrophages: Impact on the ageing central nervous system. Brain 139: 653-661, 2016.

27. Song X, Xie S, Lu K and Wang C: Mesenchymal stem cells alleviate experimental asthma by inducing polarization of alveolar macrophages. Inflammation 38: 485-492, 2015.

28. Gao S, Mao F, Zhang B, Zhang L, Zhang X, Wang M, Yan Y, Yang T, Zhang J, Zhu W, et al: Mouse bone marrow-derived mesenchymal stem cells induce macrophage M2 polarization through the nuclear factor- $\kappa \mathrm{B}$ and signal transducer and activator of transcription 3 pathways. Exp Biol Med (Maywood) 239: 366-375, 2014

29. Zhang QZ, Su WR, Shi SH, Wilder-Smith P, Xiang AP, Wong A, Nguyen AL, Kwon CW and Le AD: Human gingiva-derived mesenchymal stem cells elicit polarization of $\mathrm{m} 2$ macrophages and enhance cutaneous wound healing. Stem Cells 28: 1856-1868, 2010.

30. Ashley JW, Hancock WD, Nelson AJ, Bone RN, Tse HM, Wohltmann M, Turk J and Ramanadham S: Polarization of Macrophages toward M2 Phenotype Is Favored by Reduction in iPLA2beta (Group VIA Phospholipase A2). J Biol Chem 291: 23268-23281, 2016.

31. Geng Y, Zhang L, Fu B, Zhang J, Hong Q, Hu J, Li D, Luo C, Cui S, Zhu F and Chen X: Mesenchymal stem cells ameliorate rhabdomyolysis-induced acute kidney injury via the activation of M2 macrophages. Stem Cell Res Ther 5: 80, 2014.

32. Duffy MM, McNicholas BA, Monaghan DA, Hanley SA, McMahon JM, Pindjakova J, Alagesan S, Fearnhead HO and Griffin MD: Mesenchymal stem cells and a vitamin D receptor agonist additively suppress $\mathrm{T}$ helper 17 cells and the related inflammatory response in the kidney. Am J Physiol Renal Physiol 307: F1412-F1426, 2014.
33. Dayan V, Yannarelli G, Billia F, Filomeno P, Wang XH, Davies JE and Keating A: Mesenchymal stromal cells mediate a switch to alternatively activated monocytes/macrophages after acute myocardial infarction. Basic Res Cardiol 106: 1299-1310, 2011.

34. Krasnodembskaya A, Samarani G, Song Y, Zhuo H, Su X, Lee JW, Gupta N, Petrini M and Matthay MA: Human mesenchymal stem cells reduce mortality and bacteremia in gram-negative sepsis in mice in part by enhancing the phagocytic activity of blood monocytes. Am J Physiol Lung Cell Mol Physiol 302: L1003-L1013, 2012.

35. Xu H, Zhu J, Smith S, Foldi J, Zhao B, Chung AY, Outtz H, Kitajewski J, Shi C, Weber S, et al: Notch-RBP-J signaling regulates the transcription factor IRF8 to promote inflammatory macrophage polarization. Nat Immunol 13: 642-650, 2012.

36. Tran TH, Mattheolabakis G, Aldawsari H and Amiji M: Exosomes as nanocarriers for immunotherapy of cancer and inflammatory diseases. Clin Immunol 160: 46-58, 2015.

37. Sun W, Pang Y, Liu Z, Sun L, Liu B, Xu M, Dong Y, Feng J, Jiang C, Kong W and Wang X: Macrophage inflammasome mediates hyperhomocysteinemia-aggravated abdominal aortic aneurysm. J Mol Cell Cardiol 81: 96-106, 2015.

38. Kim OS, Seo CS, Kim Y, Shin HK and Ha H: Extracts of Scutellariae Radix inhibit low-density lipoprotein oxidation and the lipopolysaccharide-induced macrophage inflammatory response. Mol Med Rep 12: 1335-1341, 2015.

39. Sánchez-Quesada C, López-Biedma A and Gaforio JJ: Maslinic Acid enhances signals for the recruitment of macrophages and their differentiation to $\mathrm{m} 1$ state. Evid Based Complement Alternat Med 2015: 654721, 2015.

40. Németh K, Leelahavanichkul A, Yuen PS, Mayer B, Parmelee A, Doi K, Robey PG, Leelahavanichkul K, Koller BH, Brown JM, et al: Bone marrow stromal cells attenuate sepsis via prostaglandin $\mathrm{E}(2)$-dependent reprogramming of host macrophages to increase their interleukin-10 production. Nat Med 15: 42-49, 2009.

41. Herbert DR, Hölscher C, Mohrs M, Arendse B, Schwegmann A, Radwanska M, Leeto M, Kirsch R, Hall P, Mossmann H, et al: Alternative macrophage activation is essential for survival during schistosomiasis and downmodulates T helper 1 responses and immunopathology. Immunity 20: 623-635, 2004.

42. Zhou D, Huang C, Lin Z, Zhan S, Kong L, Fang C and Li J: Macrophage polarization and function with emphasis on the evolving roles of coordinated regulation of cellular signaling pathways. Cell Signal 26: 192-197, 2014.

43. Zeng KW, Song FJ, Wang YH, Li N, Yu Q, Liao LX, Jiang Y and Tu PF: Induction of hepatoma carcinoma cell apoptosis through activation of the JNK-nicotinamide adenine dinucleotide phosphate (NADPH) oxidase-ROS self-driven death signal circuit. Cancer Lett 353: 220-231, 2014.

44. Singla RD, Wang J and Singla DK: Regulation of Notch 1 signaling in THP-1 cells enhances M2 macrophage differentiation. Am J Physiol Heart Circ Physiol 307: H1634-H1642, 2014.

45. Fleming BD and Mosser DM: Regulatory macrophages: Setting the threshold for therapy. Eur J Immunol 41: 2498-2502, 2011.

46. Xu J, Chi F, Guo T, Punj V, Lee WN, French SW and Tsukamoto H: NOTCH reprograms mitochondrial metabolism for proinflammatory macrophage activation. J Clin Invest 125: 1579-1590, 2015.

47. Li B, Zhang H, Zeng M, He W, Li M, Huang X, Deng DY and Wu J: Bone marrow mesenchymal stem cells protect alveolar macrophages from lipopolysaccharide-induced apoptosis partially by inhibiting the Wnt/beta-catenin pathway. Cell Biol Int 39: 192-200, 2015

This work is licensed under a Creative Commons Attribution-NonCommercial-NoDerivatives 4.0 International (CC BY-NC-ND 4.0) License. 